\title{
Analysing impact of major solar flares on ionospheric total electron content using NavIC signals
}

\author{
Sonal Parmar ${ }^{1 \%}$, Upena Dalal ${ }^{2}$ and Kamlesh Pathak ${ }^{3}$ \\ ${ }^{1}$ Department of Electronics Engineering, Sardar Vallabhai National Institute of Technology, Surat 395007 , India and \\ M.P.S.T.M.E., Narsee Mongee Institute of Management Studies University, Mumbai 400 056, India \\ ${ }^{2}$ Department of Electronics Engineering, Sardar Vallabhai National Institute of Technology, Surat 395007 , India \\ ${ }^{3}$ Department of Applied Physics, Sardar Vallabhai National Institute of Technology, Surat 395 007, India
}

A Navigation with Indian Constellation (NavIC) receiver granted by Space Applications Centre, Ahmedabad, has been set up at SVNIT, Surat. In the present article, an effort has been made to study ionosphere response with NavIC signals during three consecutive major ' $M$ ' class and ' $C$ ' class solar flare events which took place on 22,23 and 24 July 2016. Empirical mode decomposition (EMD) and variable mode decomposition (VMD) have been used to decompose total electron content (TEC). Pearson correlation coefficients are synthesized and processed for determining the effect of solar flares on TEC data during these events. VMD gives better results compared to EMD for determining TEC fluctuations during the occurrences of solar flare events. Automatic ionospheric scintillation model (RTISM) based on Hilbert-Huang transform is applied to determine automatic threshold using Neyman Pearson detector, probability of detection and probability of false alarm using TEC.

Keywords: Empirical mode decomposition, ionospheric scintillation, NavIC, solar flares, total electron content, variable mode decomposition.

THE Navigation with Indian Constellation (NavIC) is indigenously designed by the Indian Space Research Organisation (ISRO). It provides services like navigation, positioning and precise timing in India and fifteen hundred kilometres around the Indian region ${ }^{1,2}$. NavIC uses frequencies, namely L5 $(1176.45 \mathrm{MHz})$ and $\mathrm{S}$ band (2492.028 MHz). NavIC planning mainly contains three segments, viz. space segment, ground (control) segment and consumer segment. The space division of NavIC is a constellation of seven satellites, namely IRNSS 1AIRNSS 1F (having pseudo-random number (PRN) 1-7). These numbers are allotted to numerous NavIC satellites for identification. Three satellites are placed in the Geostationary Earth Orbit (GEO) located at $32.5^{\circ}, 83^{\circ}$ and $131.5^{\circ} \mathrm{E}$ and the rest in Geosynchronous Orbit (GSO)

\footnotetext{
*For correspondence. (e-mail: sonal.parmar@nmims.edu)
}

having an inclination angle of $29^{\circ}$ through longitudinal passing at $55^{\circ}$ and $111.75^{\circ} \mathrm{E}$ (ref. 2). The control division consisting of controlling and monitoring earth stations takes care of the orbital position of all NavIC satellites. The consumer segment consisting of solo frequency receiver receives $\mathrm{L} 5$ or $\mathrm{S}$ band frequency and a twin frequency receiver accepts both the L5 and S frequencies.

Ionospheric scintillation is formed by abnormalities in electron concentration present in the ionosphere that fluctuates with respect to the local time of geographic location $^{3}$. This results in deviations in the refractive index because of small scale electron density anomalies imposed upon phase variations of NavIC signals when they propagate through the ionosphere. Ionospheric scintillations cause amplitude variations, phase fluctuations of satellite signals and if irregularities are high, it may deteriorate the carrier by noise ratio of NavIC signals. If the carrier to noise ratio falls below 20-25 Decibels per Hertz $(\mathrm{dB}-\mathrm{Hz})$, then the NavIC receiver will not be able to maintain lock with that particular satellite and it may interrupt services offered by the NavIC in future. There are various parameters important for measuring the impact of ionospheric scintillation on NavIC signals like total electron content (TEC), carrier by noise ratio $(\mathrm{C} / \mathrm{N})$, vertical total electron content (VTEC) of the satellite signals, phase scintillation index $\left(\sigma_{\phi}\right)$, amplitude scintillation index $\left(\mathrm{S}_{4}\right)$, lock time of satellite receiver and satellite elevation angle. In the present study, variations in TEC have been considered to determine the effect of ionospheric scintillation on NavIC signals. TEC is measured as the amount of electrons present in a column of ionosphere having a cross-sectional area of one square metre subjected to the path of the signal traversing to the NavIC receiver. It is measured in TEC units (TECU) ${ }^{4}$. Individual TECU is equal to $10^{16}$ number of electrons per metre square. The TEC is the factor of the ionosphere that disturbs the NavIC signals when they penetrate through the ionosphere. Several techniques have been established for calculating TEC data from the phase and amplitude of satellite signals. NavIC signals can also be utilized to calculate the TEC using dual-frequency satellite signals 5,6 .

CURRENT SCIENCE, VOL. 118, NO. 3, 10 FEBRUARY 2020 


\section{Data capturing and ionospheric TEC measurements}

The X-ray solar flux data with short-wavelength channel irradiance of wavelength $(0.05-0.4 \mathrm{~nm})$ and $0.1-0.8 \mathrm{~nm}$ are captured by GOES-15. It is the latest in a series of weather satellites to keep guard on storm development and climate conditions on earth. The data is provided by the United Kingdom Solar System Data Centre (CEDAUKSSDC). The parameters used here for calculation of TEC are provided by NavIC receiver mounted in the Department of Electronics Engineering, SVNIT, India. The geographical location of this receiver is $21^{\circ} 9^{\prime} 50.19^{\prime \prime} \mathrm{N}$ and $72^{\circ} 47^{\prime} 1.15^{\prime \prime} \mathrm{E}$. In the present study, NavIC signals have been utilized to study ionospheric response with TEC which are calculated during the three consecutive days of major solar flare events which occurred on 22, 23 and 24 July 2016. A class ' $C$ ' flare took place on 22 July 2016 and class ' $M$ ' flare took place on 23 and 24 July 2016. All the results presented here are implemented using MATLAB software. The parameters like psuedorange and frequencies of ' $\mathrm{S}$ ' and ' $\mathrm{L} 5$ ' bands provided by NavIC receiver are utilized to calculate TEC. Slant TEC is calculated using the following eq. (1) (ref. 4)

$$
\mathrm{TEC}=\frac{1}{40.3}\left(\frac{f_{1}^{2} * f_{2}^{2}}{f_{1}^{2}-f_{2}^{2}}\right)\left(P_{2}-P_{1}\right),
$$

where $f_{1}=2492.028 \mathrm{MHz}$ (S band frequency) and $f_{2}=1176.45 \mathrm{MHz}$ (L5 band frequency) and $P_{1}$ and $P_{2}$ represent pseudo ranges of the $f_{1}$ and $f_{2}$, frequencies respectively.

A solar flare is an unexpected intensity of Sun's illumination, which is observed near its outward surface ${ }^{7,8}$. The solar X-rays and ultra-violet radiations emitted during solar flares affect earth's ionosphere and may disrupt the services offered by NavIC. Various studies have examined the impact of solar flares on satellites signals using global positioning system (GPS). The solar flare effects on ionospheric TEC using GPS during solar minimum period were examined at Varanasi, which is located near equatorial ionization anomaly (EIA) crest area ${ }^{7}$. The enhancements in TEC were observed during the occurrence of solar flares with increased intensities in extreme ultraviolet (EUV) solar flux and simultaneous variations in the equatorial electrodynamics ${ }^{7}$.

Previous studies have shown sudden enhancements in ionospheric TECs during the intense solar flares ${ }^{9}$. A solar flare generates instant enhancements in ionization of the ionosphere of varying degree at various altitudes that are known as sudden ionospheric disturbances (SIDs) or ionospheric solar flare effects. Solar flares which occurred on 28 and 29 October 2003 and 4 November 2003 for Halloween events and Bastille Day events respectively have been studied using GPS ${ }^{10}$. It has been predicted that the EUV solar flux can increase the TEC of the sub-solar ionosphere through $30 \%$ in approximately 5 min. The effects of eleven ' $\mathrm{X}$ ' class solar flares which happened during 24 solar cycle period starting from 2009 to 2013 , on ionosphere at mid and low latitude region of Dibrugarh have also been studied ${ }^{11}$. The GPS-based TEC measurement was used to observe the solar flare impacts on the ionosphere. Table 1 shows classification of solar flares as per the peak range of solar flux at 100800 picometre (measured in watts per square metre).

\section{Solar flares on 22, 23 and 24 July 2016}

A strong solar flare of class $\mathrm{C}$ and a weak solar flare of class B occurred on 22 July 2016. A very strong flare of class $\mathrm{M}$ and class $\mathrm{C}$ and a weak flare of class B occurred on 23 July 2016. A strong solar flare of class M2.0 occurred on 24 July 2016 . Figures $1-6$ highlight the variations in solar flux (in weber per metre square $\left(\mathrm{Wb} / \mathrm{m}^{2}\right.$ on $Y$-axis) irradiance for both the wavelengths $(0.05-0.4 \mathrm{~nm})$ $(0.1-0.8 \mathrm{~nm})$ and TEC (in TEC units on $Y$-axis) with respect to time in minutes (Universal Time Coordinate) (on X-axis) pertaining to 22, 23 and 24 July 2016 for PRN2 and PRN3 NavIC satellite signals respectively. The vertical colour bar scale indicates intensities of solar flux irradiance and TEC increasing from bottom to top with various colours. Table 2 (also Supplementary Tables 1 and 2) represent the time of occurrences of these solar flares and corresponding TEC values (in TECU) for 22, 23 and 24 July 2016.

It is clear from Figures 1 and 2 and Table 2 that there are intensification in X-ray flux emissions during the period of the occurrence of various solar flares ${ }^{7}$. This has resulted in sudden enhancements in the ionospheric TEC values at the occurrence of each solar flares. The TEC enhancements detected could be due to the boosts in the extreme EUV radiations associated with the solar flare and also due to the changes in the overall ionospheric dynamics and electrodynamics ${ }^{7}$. Figures 3 and 4 and Supplementary Table 1 also show that there were TEC fluctuations during the peak deviations in the solar flux irradiance. Similar observations were found for TEC fluctuation on 24 July 2016 (Figures 5 and 6 and Supplementary Table 2).

Table 1. Classification of various solar flares

\begin{tabular}{lc}
\hline Solar flare class & $\begin{array}{c}\text { Peak X-ray flux range at 100-800 picometre } \\
\text { (watts/square metre) }\end{array}$ \\
\hline Class A & $<10^{-7}$ \\
Class B & $10^{-7}-10^{-6}$ \\
Class C & $10^{-6}-10^{-5}$ \\
Class M & $10^{-5}-10^{-4}$ \\
Class X & $>10^{-4}$ \\
\hline
\end{tabular}


Table 2. Various classes of solar flares and corresponding TEC values (in TECU) on 22 July 2016

\begin{tabular}{|c|c|c|c|c|c|c|}
\hline \multirow{2}{*}{$\begin{array}{l}\text { Class of flare } \\
\text { (solar flare } \\
\text { sun spot region } \\
\text { is } 2567 \text { ) }\end{array}$} & \multirow{2}{*}{$\begin{array}{l}\text { Start time of } \\
\text { solar flare } \\
\text { (h) }\end{array}$} & \multirow{2}{*}{$\begin{array}{l}\text { Maximum } \\
\text { intensity of solar } \\
\text { flare at time } \\
\text { (h) }\end{array}$} & \multirow{2}{*}{$\begin{array}{l}\text { End time of } \\
\text { solar flare } \\
\text { (h) }\end{array}$} & \multirow{2}{*}{$\begin{array}{l}\text { Solar flux irradiance } \\
(0.05-0.4 \mathrm{~nm}) \\
\text { maximum intensity } \\
\left(\mathrm{Wb} / \mathrm{m}^{2}\right)\end{array}$} & \multicolumn{2}{|c|}{$\begin{array}{c}\text { Observations in TEC } \\
\text { (in TECU) fluctuations } \\
\text { during solar flares instances } \\
\text { during maximum intensity } \\
\text { of solar flux irradiance of } \\
0.05-0.4 \mathrm{~nm} \text { wavelength }\end{array}$} \\
\hline & & & & & PRN2 & PRN3 \\
\hline C6.6 & 06:34 (394 min) & $06: 51(411 \mathrm{~min})$ & $06: 58$ (418 min) & $1.24 \mathrm{E}-06$ & 71 & 62 \\
\hline $\mathrm{C} 1.2$ & $07: 23$ (443 $\mathrm{min})$ & $07: 26(446 \mathrm{~min})$ & 07:31 (451 min) & $8.00 \mathrm{E}-08$ & 56.80 & 47.09 \\
\hline B6.8 & $08: 30(510 \mathrm{~min})$ & $08: 34(514 \mathrm{~min})$ & 08:38 (518 min) & $2.69 \mathrm{E}-08$ & 40.17 & 28.82 \\
\hline B9.1 & $11: 14(674 \mathrm{~min})$ & $11: 31(691 \mathrm{~min})$ & $11: 45(705 \mathrm{~min})$ & $5.39 \mathrm{E}-08$ & 40.50 & 48 \\
\hline $\mathrm{C} 2.4$ & 20:09 (1209 $\mathrm{min})$ & $20: 20(1220 \mathrm{~min})$ & $20: 31(1231 \mathrm{~min})$ & $3.58 \mathrm{E}-07$ & 13.82 & 11.91 \\
\hline B5.6 & $22: 26(1346 \mathrm{~min})$ & $22: 30(1350 \mathrm{~min})$ & $22: 34(1354 \mathrm{~min})$ & $2.81 \mathrm{E}-08$ & 2.33 & 1.8 \\
\hline B7.6 & 23:09 (1389 $\mathrm{min})$ & $23: 15(1395 \mathrm{~min})$ & $23: 27(1407 \mathrm{~min})$ & $5.71 \mathrm{E}-08$ & 1.94 & 1.94 \\
\hline
\end{tabular}
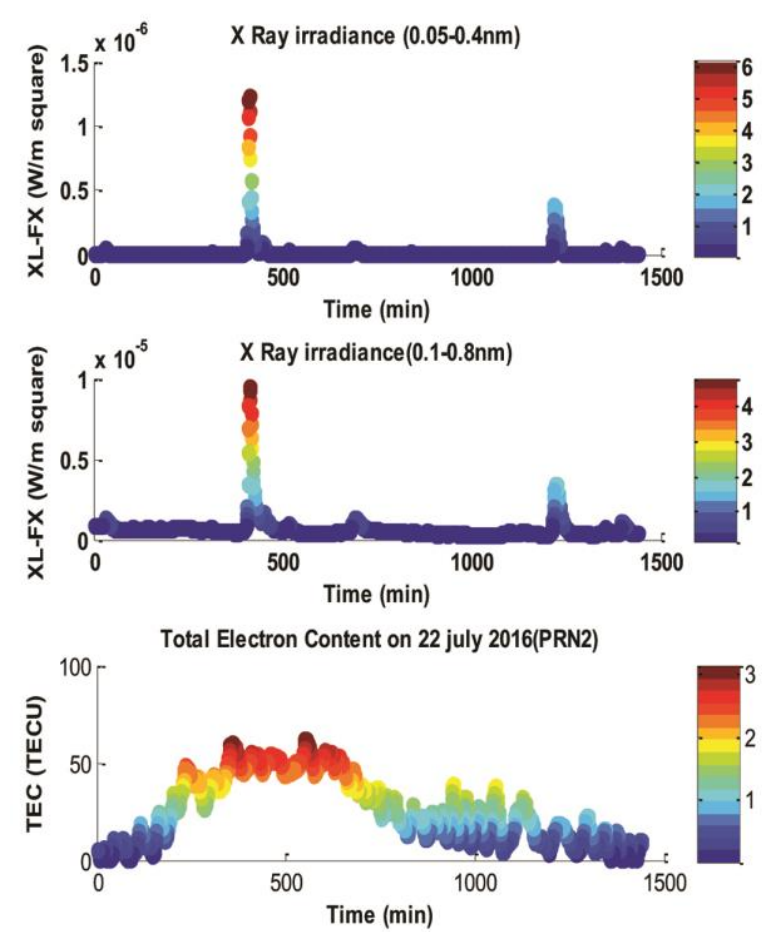

Figure 1. Solar flux irradiance $(0.05-0.4 \mathrm{~nm})(0.1-0.8 \mathrm{~nm})$ and TEC variations for PRN2 on 22 July 2016.

Earlier studies have shown that the maximum value of the rate of change of TEC depends on the flare class and rate of change in the flare radiations ${ }^{12}$. The main abnormalities from the mean TEC may be additionally due to the complete variations in the low latitude electrodynamics, like fountain effect and the meridional wind effects $^{7,13}$. It is observed from the study of effects of solar flares of 22, 23 and 24 July 2016 on ionospheric TEC, that there are enhancements in TEC for major solar flare events like class $M$ and class $C$ and drop in TEC values for weak solar flares. Hence it is clear that a substantial boost in TEC was observed in this equatorial anomaly region (EIA) during the occurrences of each class of solar
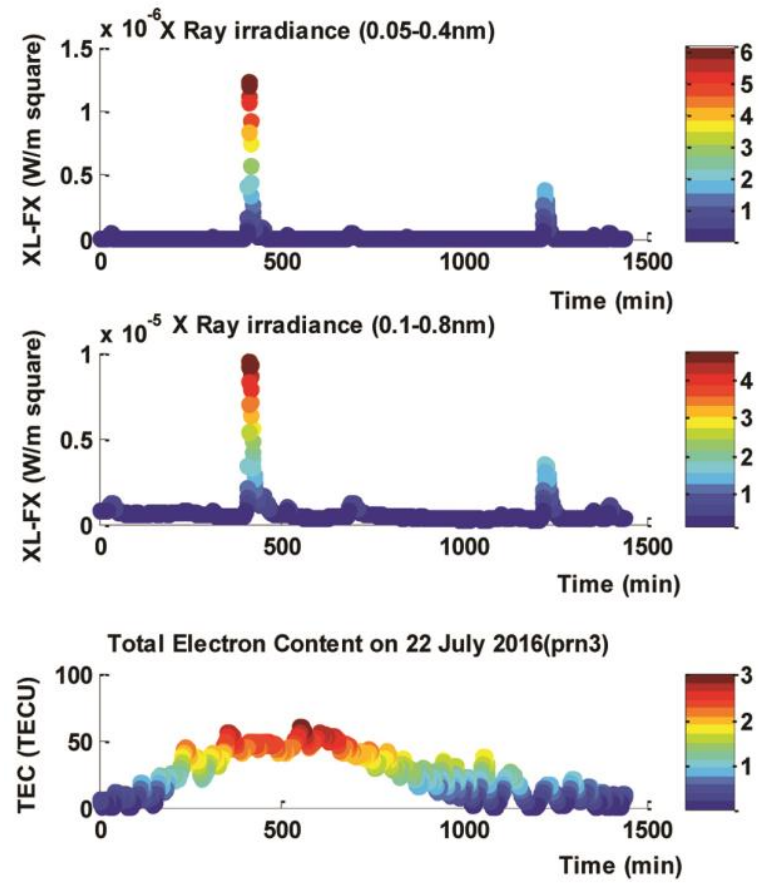

Figure 2. Solar flux irradiance $(0.05-0.4 \mathrm{~nm})(0.1-0.8 \mathrm{~nm})$ and TEC variations for PRN3 on 22 July 2016.

flares with simultaneous enhancements in ionization ${ }^{7,8}$. The present study is significant as it attempts to utilize the NavIC signals to study the impact of class M, C and B solar flares which occurred on three consecutive days of 22, 23 and 24 July 2016, on ionospheric TEC in this region.

\section{Examining TEC with VMD and EMD techniques}

The adaptive data analysis techniques like empirical mode decomposition (EMD) and Hilbert spectral analysis (HSA) are efficiently-developed methods which can be

CURRENT SCIENCE, VOL. 118, NO. 3, 10 FEBRUARY 2020 
used for geophysical studies ${ }^{14}$. The data from the natural phenomenon are normally nonstationary because of their temporary nature. The capture time of data may also be lesser than the time scale length that states the phenome-

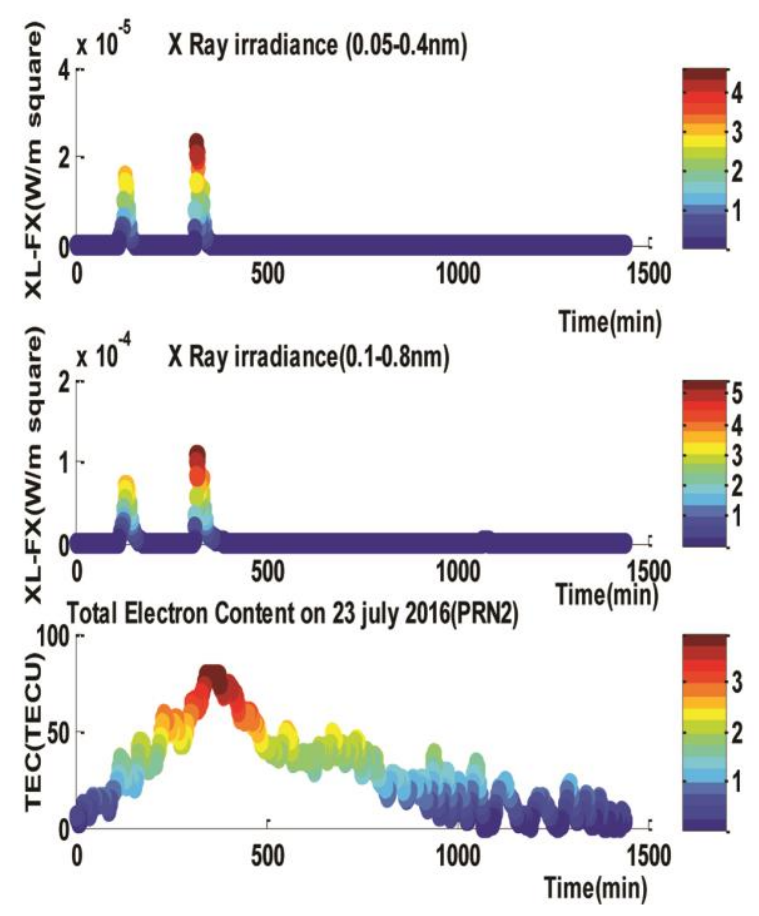

Figure 3. Solar flux irradiance $(0.05-0.4 \mathrm{~nm})(0.1-0.8 \mathrm{~nm})$ and TEC variations for PRN2 on 23 July 2016.

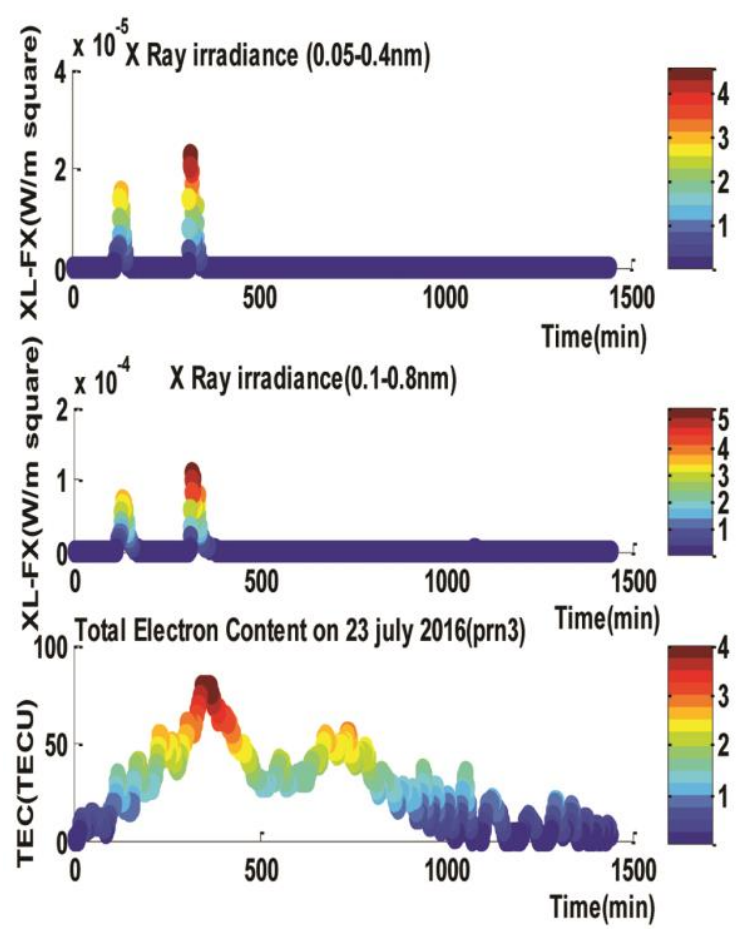

Figure 4. Solar flux irradiance $(0.05-0.4 \mathrm{~nm})(0.1-0.8 \mathrm{~nm})$ and TEC variations for PRN3 on 23 July 2016. non. The Hilbert-Huang transform (HHT) is suitable for treating nonlinear and nonstationary signals ${ }^{14,15}$. The EMD technique decays the input signal into its intrinsic mode functions (IMFs) which consist of sharp instantaneous frequencies as explained in refs 14,15 . The breakdown is done on the local period of the input signal in time domain. Each IMF should achieve the subsequent
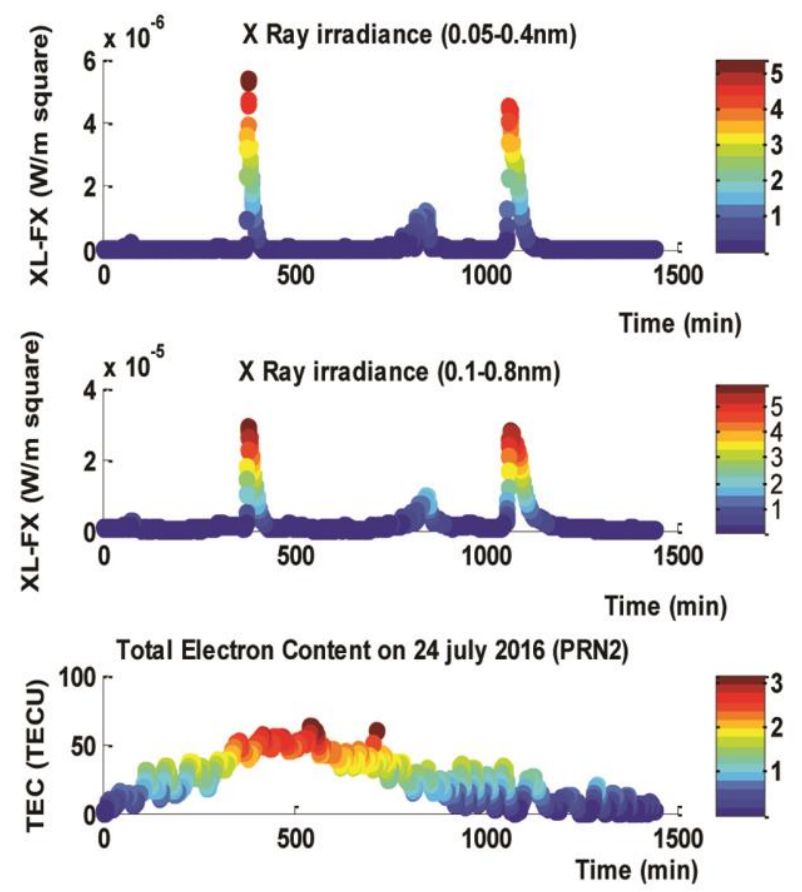

Figure 5. Solar flux irradiance $(0.05-0.4 \mathrm{~nm})(0.1-0.8 \mathrm{~nm})$ and TEC variations for PRN2 on 24 July 2016.
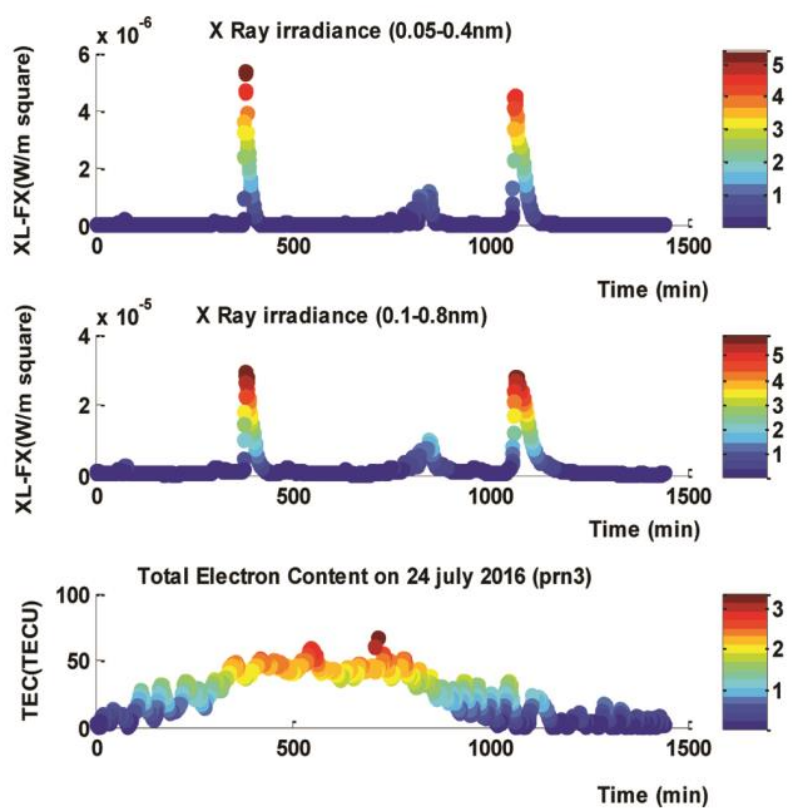

Figure 6. Solar flux irradiance $(0.05-0.4 \mathrm{~nm})(0.1-0.8 \mathrm{~nm})$ and TEC variations for PRN3 on 24 July 2016. 
two necessities: (i) In the entire data, the numeral of extrema and the occurrences of zero crossings must be identical, or they can vary only by one. (ii) At any data point, the mean value of the envelope which is defined using local maxima and local minima should be zero. An IMF is any function with a similar number of extrema and zero crossings, with its envelopes being symmetric with respect to zero ${ }^{14-16}$. Here the 'S stoppage criterion' is used to stop the EMD decomposition process ${ }^{16}$. By using this criterion, the shifting process stops only if the numbers of zero crossings and extrema are equal or at most differ by one and stay the same for ' $S$ ' consecutive times. In the present analysis, the number of intrinsic mode functions generated in a set depends upon input TEC. The EMD algorithms are given in appendices 1 and 2 (refs 14,15$)$.

\section{VMD technique}

EMD has some restrictions such as sensitivity to noise and sampling ${ }^{17}$. These limitations can be overcome through the recently developed non-recursive variable mode decomposition model in which the modes are derived simultaneously. This model creates an ensemble of modes and their particular centre frequencies in such a manner that the modes together replicate the input signal and then result into baseband signal after demodulation. VMD model is basically a spectrum-based decomposition technique of a one-dimensional input signal into ' $k$ ' band separated modes. This model can be efficiently enhanced using an alternating direction method of multipliers technique $^{17,18}$. This model is the most effective way of handling non-stationary signals. Various studies have compared analysis of VMD and EMD techniques ${ }^{18-21}$. However, earlier studies mainly focused on relating the time-varying waveforms of the signals decayed respectively by both the techniques rather than determining noise robustness and capabilities of the VMD model in determining time-frequency features from non-stationary signals. In this article, VMD and EMD techniques have been deployed for detecting ionospheric irregularities using TEC for NavIC signals in a low latitude region during solar flare events ${ }^{18}$.

The important parameters for VMD are (i) the number of modes ' $\mathrm{K}$ ' which are found on the basis of the number of frequency constituents in the input signal and (ii) bandwidth control parameter $\alpha$, which is found based on the centre frequency of importance. Both these parameters are interrelated to each other ${ }^{18}$.

\section{Results and discussions}

The EMD and VMD algorithms have been used for decomposing TEC data determined on 22, 23 and 24 July 2016 during the solar flare events. The fluctuations in
TEC can be predicted with high resolutions when we decompose it into mode functions. The EMD decomposition of TEC was accomplished to recognize and determine the intensity of scintillating signals during these events. A major solar flare event of class $M$ and class $C$ took place on these days. Figures 7 and 8 represent EMD and VMD decomposition of TEC data on 22 July 2016 for PRN2 IRNSS satellite respectively. The primary IMF contains the maximum frequency and then frequency declines for the following IMFs. These IMFs possess the characteristics of the original input TEC signal. The low order IMFs

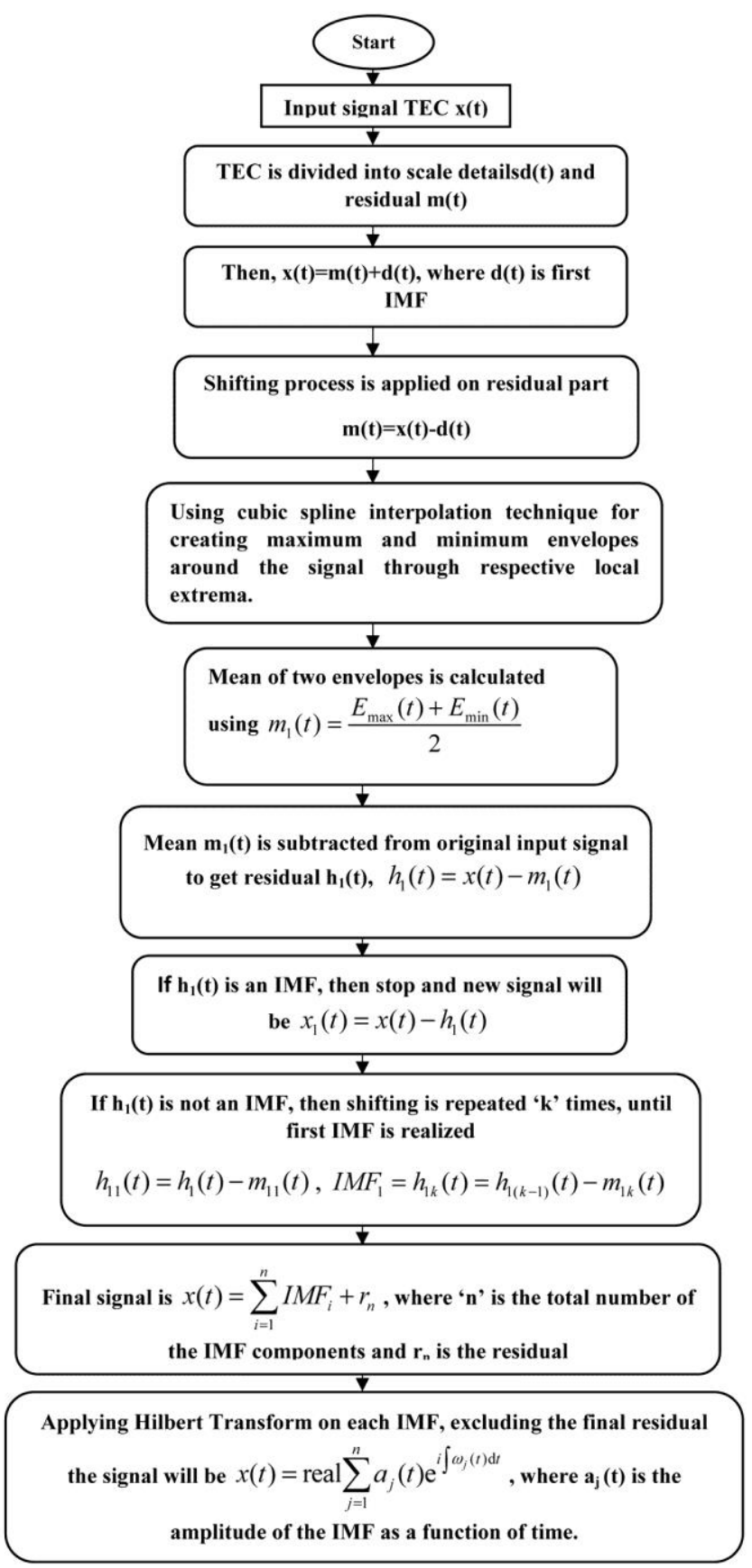

Appendix 1.

CURRENT SCIENCE, VOL. 118, NO. 3, 10 FEBRUARY 2020 
1,2 and 3 is a representation of noise in the input measured TEC. They do not contain information related to the presence of the disturbances due to solar flare events and hence can be neglected. The IMFs 4 to 6 consist of oscillatory modes corresponding to the enhanced intensities of solar flux during the occurrences of solar flare event. These subset of components demonstrate mid-term variations of the TEC measurements that are correlated with

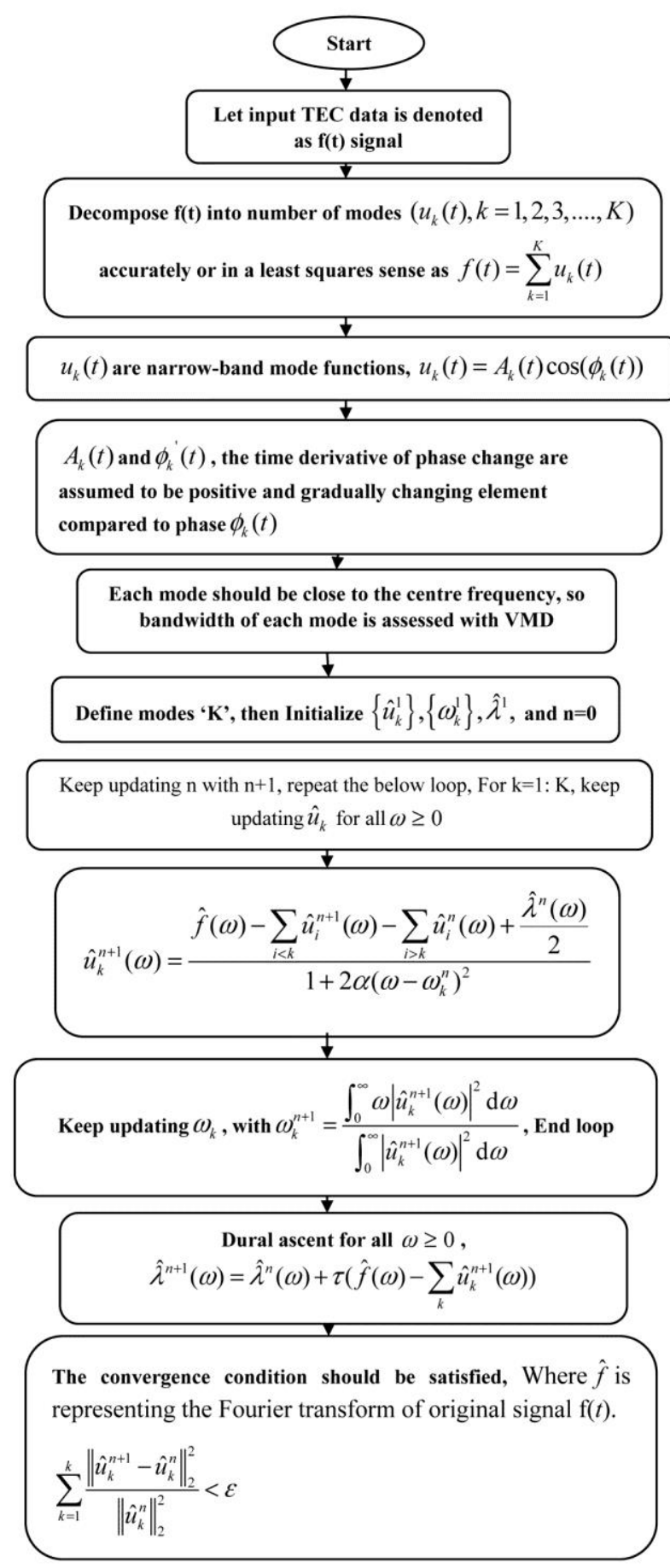

Appendix 2.
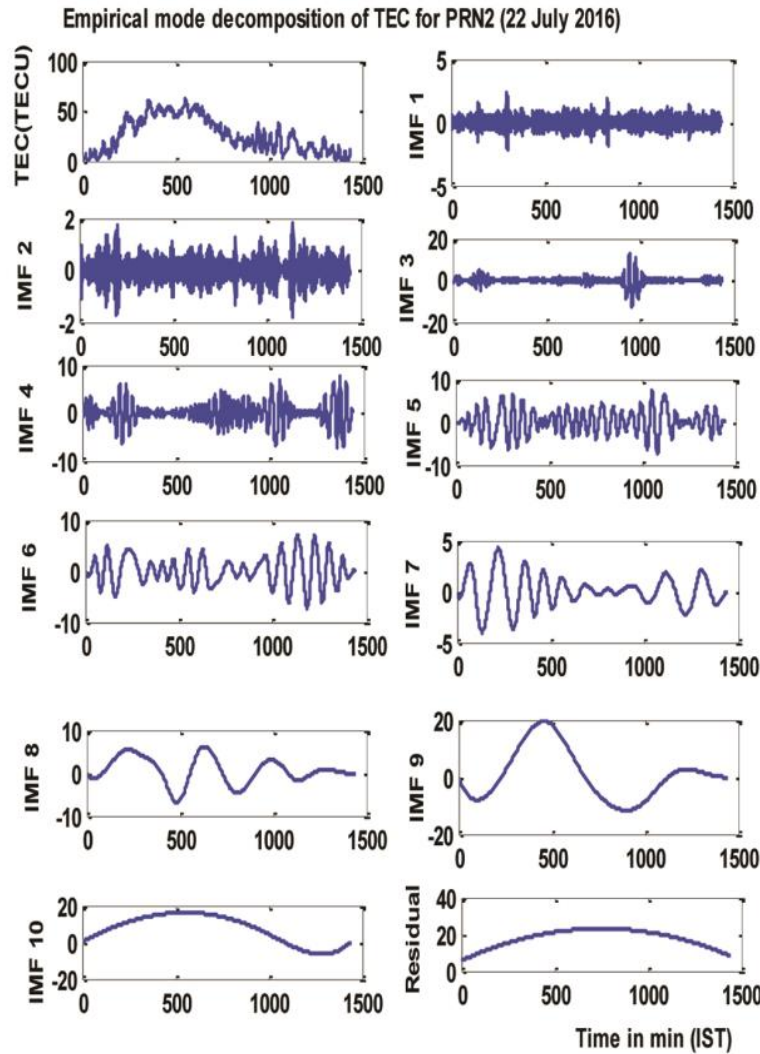

Figure 7. Empirical mode decomposition of TEC data for PRN2 on 22 July 2016.

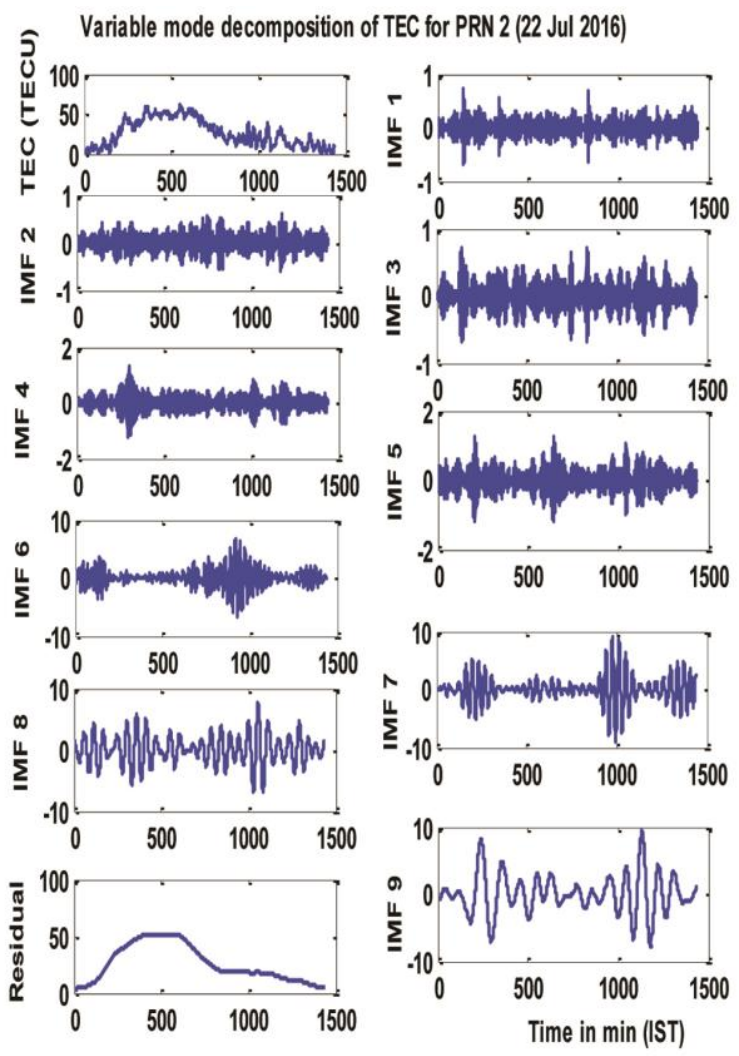

Figure 8. Variable mode decomposition of TEC data for PRN2 on 22 July 2016. 


\section{RESEARCH ARTICLES}

the physical properties of the ionospheric propagation. The IMF 7 to IMF 10 and the residual represent longterm fluctuations in measured TEC during the solar flare events. In Figure 7, it can be seen that input TEC is decomposed from IMF 1 up to IMF 10 and then residual remains. From Figure 8 it can be seen that VMD is capable of decomposing TEC with good resolution from IMF 1-IMF 9 and residual. EMD and VMD decomposition results of TEC for PRN2 and PRN3 for three consecutive days on 22, 23 and 24 July 2016 are given in Supplementary Figures $1-10$.

Fluctuations are emerged in TEC during occurrences of various classes of solar flares and it can be predicted in the layers 5 and 6 of decomposition.

\section{RTISM-HHT model for correlation analysis}

In this article an automatic ionospheric scintillation model known as RTISM has also been utilized for determining ionospheric behaviour during the three major solar flare events ${ }^{22,23}$. This model is used for determining automatic threshold using the Neyman-Pearson detec$\operatorname{tor}^{22,23}$. Earlier this model was utilized for analysing ionospheric scintillation effects on amplitude scintillation index $\left(\mathrm{S}_{4}\right)$ and phase scintillation index $\left(\sigma_{\phi}\right)$ during the geomagnetic storms that occurred in the maximum solar activity periods of the 24 solar cycle (2013-14) using GPS station 6 receiver $^{24}$. This RTISM-HHT model is used to determine the Pearson correlation coefficient (PCC) between various mode functions which has resulted due to the application of EMD and VMD techniques and the TEC. This model is also applied to extract the probability of false alarm, probability of detection and probability of missed detection ${ }^{24}$.

\section{Pearson correlation coefficient}

The Neyman-Pearson detectors are useful for determining the threshold value and they can attain extreme recognition power for a precise false alarm limit even when enough earlier data is unobtainable. The PCC is a factor to measure the relationship between two variables. The PCC is computed between TEC and IMFs and it is useful to obtain information about which IMF is relevant to $\mathrm{TEC}^{24}$. The PCC is defined as

$$
\mathrm{PCC}=\frac{\sum_{i=1}^{n}\left(x_{i}-\bar{x}\right)-\left(y_{i}-\bar{y}\right)}{\sqrt{\left[\sum_{i=1}^{n}\left(x_{i}-\bar{x}\right)^{2}\right]\left[\sum_{i=1}^{n}\left(y_{i}-\bar{y}\right)^{2}\right]} .}
$$

In the above equation, $x_{i}$ and $y_{i}$ denote the two variables, $\bar{x}$ and $\bar{y}$ represent the mathematical mean of the vari- ables $x_{i}$ and $y_{i}, n$ represents the sample size of the variables $x_{i}$ and $y_{i}$. The $x_{i}$ is the input TEC and $y_{i}$ is the intrinsic mode functions resulting with EMD and VMD techniques.

\section{EMD and detecting threshold}

The TEC data is first decayed into a finite set of mode functions using EMD and VMD techniques. The PCC of the resultant mode functions is then determined. The threshold level is derived using eq. (3) ${ }^{24}$. If the PCC of the IMFs is $\mu_{i}$ and the threshold is represented as $\tau$, the equation for the threshold is given as

$$
T=\left[\frac{\log _{e} \lambda}{\mu}+\frac{\mu}{2}\right] .
$$

The relevant IMFs are selected on the basis of their coefficient values calculated earlier in comparison to the threshold value. If $\mu_{i} \geq \tau$, the IMF is relevant and if the condition is not satisfied, it is irrelevant. The probability of false alarm can be determined using eq. (4) $)^{24,25}$

$$
p_{f a}=\operatorname{erfc}\left[\frac{\log _{e} \lambda}{\mu}+\frac{\mu}{2}\right] .
$$

The probability of detection is determined using eq. $(5)^{24}$

$$
P_{D}=\operatorname{erfc}\left[\frac{\log _{e} \lambda}{\mu}-\frac{\mu}{2}\right] .
$$

The probability of missed detection is calculated using eq. $(6)^{24}$

$$
P_{\text {miss }}=1-P_{D}
$$

Table 3 highlights the computed results of PCC, average of PCC, probability of false alarm, probability of detection and probability of missed detection for PRN2 and PRN3 IRNSS satellites on 22, 23 and 24 July 2016 respectively. It is clear from the Table 3, that the PCC determined using VMD model is giving better results compared with EMD results. The PCC between IMF 10 and input TEC is 0.95 for PRN2 and PRN3 on 22 July 2016. Similarly, PCC between the IMF 10 and input TEC is 0.96 and 0.91 for PRN2 and PRN3 respectively on 23 July 2016. Moreover, PCC between the IMF 10 and input TEC signal is 0.95 for PRN2 and PRN3 on 24 July 2016. The probability of false alarm is -0.5 using EMD model and 0.5 using VMD model for PRN2 on 22 July 2016. The probability of false alarm is 0.32 using EMD model and 0.5 using VMD model for PRN3 on 22 July 2016. The probability of false alarm is 0.5 using EMD and VMD model for PRN2 and PRN3 on 23 July 2016. The 
RESEARCH ARTICLES

Table 3. EMD and VMD analysis parameters for PRN2 and PRN3 on 22, 23, 24 July 2016

\begin{tabular}{|c|c|c|c|c|c|c|c|c|c|c|c|c|}
\hline \multirow{3}{*}{$\begin{array}{l}\text { Day } \\
\text { PCC } \\
\text { Mode functions }\end{array}$} & \multicolumn{4}{|c|}{22 July 2016} & \multicolumn{4}{|c|}{23 July 2016} & \multicolumn{4}{|c|}{24 July 2016} \\
\hline & \multicolumn{2}{|c|}{ PCC for PRN2 } & \multicolumn{2}{|c|}{ PCC for PRN3 } & \multicolumn{2}{|c|}{ PCC for PRN2 } & \multicolumn{2}{|c|}{ PCC for PRN3 } & \multicolumn{2}{|c|}{ PCC for PRN2 } & \multicolumn{2}{|c|}{ PCC for PRN3 } \\
\hline & EMD & VMD & EMD & VMD & EMD & VMD & EMD & VMD & EMD & VMD & EMD & VMD \\
\hline 1 & 0.03 & 0.02 & 0.03 & 0.02 & 0.01 & 0.01 & 0.03 & 0.01 & 0.04 & 0.02 & 0.04 & 0.02 \\
\hline 2 & 0.02 & 0.02 & 0.04 & 0.02 & 0.02 & 0.01 & 0.02 & 0.01 & 0.03 & 0.02 & 0.02 & 0.02 \\
\hline 3 & 0.16 & 0.02 & 0.12 & 0.02 & 0.06 & 0.01 & 0.17 & 0.02 & 0.16 & 0.02 & 0.03 & 0.02 \\
\hline 4 & 0.18 & 0.03 & 0.11 & 0.02 & 0.15 & 0.02 & 0.14 & 0.02 & 0.17 & 0.03 & 0.20 & 0.03 \\
\hline 5 & 0.19 & 0.04 & 0.26 & 0.03 & 0.18 & 0.02 & 0.18 & 0.03 & 0.18 & 0.03 & 0.18 & 0.03 \\
\hline 6 & 0.16 & 0.07 & 0.07 & 0.06 & 0.23 & 0.04 & 0.12 & 0.06 & 0.20 & 0.04 & 0.14 & 0.04 \\
\hline 7 & 0.14 & 0.14 & 0.00 & 0.13 & 0.06 & 0.10 & 0.13 & 0.12 & 0.07 & 0.08 & 0.13 & 0.07 \\
\hline 8 & 0.10 & 0.19 & -0.05 & 0.18 & 0.33 & 0.16 & 0.22 & 0.17 & -0.11 & 0.17 & 0.02 & 0.15 \\
\hline 9 & 0.79 & 0.26 & 0.18 & 0.26 & 0.90 & 0.25 & 0.00 & 0.57 & 0.67 & 0.26 & 0.02 & 0.26 \\
\hline 10 & 0.56 & 0.95 & 0.03 & 0.95 & 0.01 & 0.96 & 0.03 & 0.91 & 0.04 & 0.95 & 0.04 & 0.95 \\
\hline Average & 0.27 & 0.18 & 0.15 & 0.17 & 0.25 & 0.16 & 0.15 & 0.19 & 0.17 & 0.16 & 0.12 & 0.16 \\
\hline Threshold & -0.01 & 0.00 & 0.01 & 0.00 & -0.01 & 0.00 & 0.00 & 0.00 & 0.02 & 0.00 & -0.01 & -0.01 \\
\hline $\begin{array}{c}\text { Probability of } \\
\text { false alarm }\end{array}$ & -0.50 & 0.50 & 0.32 & 0.50 & 0.50 & 0.50 & 0.50 & 0.50 & 0.49 & 0.50 & 0.50 & 0.50 \\
\hline $\begin{array}{c}\text { Probability of } \\
\text { detection }\end{array}$ & 1 & 1 & 1 & 1 & 1 & 1 & 1 & 1 & 1 & 1 & 1 & 1 \\
\hline $\begin{array}{l}\text { Probability of missed } \\
\text { detection }\end{array}$ & 0 & 0 & 0 & 0 & 0 & 0 & 0 & 0 & 0 & 0 & 0 & 0 \\
\hline
\end{tabular}

probability of false alarm is 0.49 using EMD model and 0.5 using VMD model for PRN2 on 24 July 2016. Probability of false alarm is 0.5 using EMD and VMD model for PRN3 on 24 July 2016. The probability of detection is equivalent to 1 for PRN2 and PRN3 IRNSS satellites on all the three days, thus denoting the probability of missed detection equal to zero. The PCC values larger than the predictable threshold help in determining the relevant IMFs for each satellite.

\section{Conclusions}

The ionospheric TEC has been determined during the consecutive three days of major solar flares which occurred on 22, 23 and 24 July 2016 with NavIC receiver set up at SVNIT, India. This NavIC helps in the study of ionosphere and for evaluating the performance of NavIC receiver during various natural phenomena. The variations in TEC have also been studied during occurrences of various classes of solar flares on these three days with respect to X-ray solar flux. The X-ray solar flux data with short-wavelength channel irradiance of wavelength $(0.05-0.4 \mathrm{~nm})$ and $(0.1-0.8 \mathrm{~nm})$ are provided by GOES 15 (data provided by CEDA-UKSSDC). The EMD and VMD techniques have been utilized for decomposing TEC data and precisely locating instances of occurrences of various solar flares. The PCC, probability of false alarm, probability of detection and probability of missed detection have also been calculated using the RTISMHHT model. It is clear from the RTISM-HHT model for correlation analysis that VMD gives better results compared to EMD. The present results can be used for deter- mining fluctuations in TEC during the other atmospheric phenomenon or more number of solar flare events.

1. Yashaswini, A. R., Reddy, P. S. N. and Ramaiah, G. N. K., Generation and implementation of IRNSS standard positioning signal. Eng. Sci. Technol., 2016, 19(3), 1381-1389.

2. Vasudha, M. P. and Raju, G., Comparative evaluation of IRNSS performance with special reference to positional accuracy. Gyrosc. Navigation, 2017, 8(2), 136-149.

3. Zou, Y. and Wang, D., A study of GPS ionospheric scintillations observed at Guilin. J. Atmos. Solar-Terrest. Phys., 2009, 71(1718), 1948-1958.

4. Norsuzila, Y., Abdullah, M., Ismail, M., Ibrahim, M. and Zakaria, Z., Total electron content (TEC) and estimation of positioning error using Malaysia data. Proceedings of the World Congress on Engineering Vol. I WCE 2010, London, UK, 30 June-2 July 2010.

5. Warnant, R. and Pottiaux, E., The increase of the ionospheric activity as measured by GPS. Earth Planets Space, 2000, 52(11), 1055-1060.

6. Arikan, F., Arikan, O. and Erol, C. B., Regularized estimation of TEC from GPS data for certain midlatitude stations and comparison with the IRI model. Adv. Space Res., 2007, 39(5), 867-874.

7. Kumar, S. and Singh, A. K., Effect of solar flares on ionospheric TEC at Varanasi, near EIA crest during solar minimum period. Indian J. Radio Space Phys., 2012, 41, 141-147.

8. Huijun, L., Libo, L., Bin, C., Jiuhou, L., Xinan, Y. and Weix, W., Modeling the response of middle latitude ionosphere to solar flares. J. Atmos. Solar-Terrest. Phys., 2007, 69, 1587-1598.

9. Weixing, W., Libo, L., Hong, Y., Baiqi, N. and Shunrong, Z., The GPS measured SITEC caused by the very intense solar flare on 14 July 2000. Adv. Space Res., 2005, 36, 2465-2469.

10. Tsurutani, B. T., Verkhoglyadova, O. P., Mannucci, A. J., Lakhina, G. S., Li, G. and Zank, G. P., A brief review of solar flare effects on the ionosphere. Radio Sci., 2009, 44, 1-14.

11. Hazarika, R., Kalita, B. R. and Bhuyan, P. K., Ionospheric response to X-class solar flares in the ascending half of the subdued solar cycle 24. J. Earth Syst. Sci., 2016, 125(6), 1235-1244. 
12. Liu, J. Y., Lin, C. H., Tsai, H. F. and Liou, Y. A., Ionospheric solar flare effects monitored by the ground-based GPS receivers: Theory and observation. J. Geophys. Res. USA, 2004, 109, A01307.

13. Manju, G., Pant, T. K. and Devasia, C. V., Ravindran, S. and Sridharan, S., Electrodynamical response of the Indian low-mid latitude ionosphere to the very large solar flare of 28 October $2003-$ a case study. Ann. Geophys. (Germany), 2009, 27, 3853-3860.

14. Huang, N. E. and Zhaohua, Wu., A review on Hilbert-Huang transform method and its applications to geophysical studies. Rev. Geophys., 2008, 46(2), 1944-9208.

15. Huang, N. E. et al., The empirical mode decomposition and the Hilbert spectrum for nonlinear and nonstationary time series analysis. Proc. R. Soc. London, Ser. A, 1998, 454, 903-993.

16. Huang, N. E., Wu, M. L., Long, S. R., Shen, S. S., Qu, W. D. Gloersen, P. and Fan, K. L., A confidence limit for the position empirical mode decomposition and Hilbert spectral analysis. Proc. R. Soc. London, Ser. A, 2003, 459, 2317-2345.

17. Dragomiretskiy, K. and Zosso, D., Variational mode decomposition. IEEE Trans. Signal Process., 2014, 62(3), 531-544.

18. Yang, W., Peng, Z., Wei, K., Shi, P. and Tian, W., Superiorities of variational mode decomposition over empirical mode decomposition particularly in time-frequency feature extraction and wind turbine condition monitoring. IET Renewable Power Generation, 2017, 11(4), 443-452.

19. Lv, Z., Tang, B., Zhou, Y. and Zhou, C., A novel method for mechanical fault diagnosis based on variational mode decomposition and multikernel support vector machine. Shock Vibr., 2016, art.Id. 3196465, 1-11.

20. Lahmiri, S., Comparing variational and empirical mode decomposition in forecasting day-ahead energy prices. IEEE Syst. J., 2016, 99, $1-4$.
21. An, X. and Zeng, H., Pressure fluctuation signal analysis of a hydraulic turbine based on variational mode decomposition. $J$. Power Energy, 2015, 229(8), 978-991.

22. Qin, Z., Chen, H. and Chang, J., Signal-to-noise ratio enhancement based on empirical mode decomposition in phase sensitive optical time domain reflectometry systems. Sensors, 2017, 1-10.

23. Ayenu-Prah, A. and Attoh-Okine, N., A criterion for selecting relevant intrinsic mode functions in empirical mode decomposition. Adv. Adapt. Data Anal. Theory Appl., 2010, 2(1), 1-24.

24. Ratnam, D. V., Sivavaraprasad, G. and Lee, J., Automatic ionospheric scintillation detector for global navigation satellite system receivers. IET Radar, Sonar Navigation, 2015, 9(6), 702-711.

25. Kolawole, M. O., Radar Systems, Peak Detection and Tracking, Elsevier, 2002.

ACKNOWLEDGEMENTS. We thank the Space Applications Centre, Ahmedabad for granting and installing Navigation with Indian Constellation (NavIC) receiver in the Department of Electronics Engineering at Sardar Vallabhai National Institute of Technology (SVNIT) and S.P. thanks the United Kingdom Solar System Data Centre (CEDAUKSSDC) for providing solar flux irradiance data provided by Geostationary Operational Environmental Satellite (GOES-15) spacecraft. The NavIC receiver at Department of Electronics Engineering, SVNIT, India, is being administered by Dr Upena Dalal.

Received 17 December 2018; revised accepted 14 October 2019

doi: $10.18520 / \mathrm{cs} / \mathrm{v} 118 / \mathrm{i} 3 / 392-400$ 